\title{
Comparison of the Respiratory Tractmicrobiome in Hospitalized COVID-19 Patients with Different Disease Severity
}

\section{Jiali Chen}

Chinese PLA Center for Disease Control and Prevention

\section{Xiong Liu}

Chinese PLA Center for Disease Control and Prevention

Wei Liu

Chinese PLA Center for Disease Control and Prevention

\section{Chaojie Yang}

Chinese PLA Center for Disease Control and Prevention

\section{Ruizhong Jia}

Chinese PLA Center for Disease Control and Prevention

\section{Yuehua Ke}

Chinese PLA Center for Disease Control and Prevention

\section{Jinpeng Guo}

Chinese PLA Center for Disease Control and Prevention

\section{Leili Jia}

Chinese PLA Center for Disease Control and Prevention

\section{Changjun Wang}

Chinese PLA Center for Disease Control and Prevention

Yong Chen ( $\square$ chenyonger@126.com)

Chinese PLA Center for Disease Control and Prevention

\section{Research Article}

Keywords: COVID-19, respiratory tract microbiome, disease severity, biomarker

Posted Date: January 12th, 2022

DOI: https://doi.org/10.21203/rs.3.rs-1237564/v1

License: (1) (1) This work is licensed under a Creative Commons Attribution 4.0 International License.

Read Full License 


\section{Abstract}

Background: Little is known about the characteristics of respiratory tract microbiome in Coronavirus disease 2019 (COVID-19) inpatients with different severity.

Methods: A cross-sectional study was conducted to characterize respiratory tract microbial communities of 69 COVID-19 inpatients from 64 nasopharyngeal swabs and 5 sputum specimens using 16S ribosomal RNA (rRNA) gene V3-V4 region sequencing. The bacterial profiles were used to find potential biomarkers by the two-step method, the combination of random forest model and the linear discriminant analysis effect size (LEfSe), and explore the connections with clinical characteristics by Spearman's rank test.

Results: Compared with mild COVID-19 patients, severe patients had significantly decreased bacterial diversity ( $P$ values were less than 0.05 in the alpha and beta diversity) and relative lower abundance of opportunistic pathogens, including Actinomyces, Prevotella, Rothia, Streptococcus, Veillonella. Eight potential biomarkers including Treponema, Lachnoanaerobaculum, Parvimonas, Selenomonas, Alloprevotella, Porphyromonas, Gemellaand Streptococcus were found to distinguish the mild COVID-19 patients from the severe COVID-19 patients. The genera of Actinomyces andPrevotella were negatively correlated with age and inpatient days. Intensive Care Unit (ICU) admission, neutrophil count (GRA) and lymphocyte count (LYMPH) were significantly correlated with different genera in the two groups. In addition, there were a positive correlation between Klebsiella and white blood cell count (WBC) in two groups.

Conclusion: The respiratory tract microbiome had significant difference in COVID-19 patients with different severity. The value of the respiratory tract microbiome as predictive biomarkers for COVID-19 severity merits further exploration.

\section{Background}

As an emerging infectious disease caused by severe acute respiratory syndrome coronavirus 2 (SARSCoV-2) [1], coronavirus disease 2019 (COVID-19) has spread throughout the world, causing more than 223 million confirmed cases and over 4.6 million deaths[2]. Since the World Health Organization (WHO) has declared COVID-19 outbreak a global pandemic on March 11, 2020[3], the pandemic has already brought an enormous threat to the global economy and public health burden.

SARS-CoV-2 may damage the epithelial barrier acutely and promote invasions of other pathogens[4]. Moreover, viral respiratory infections such as influenza virus and human coronavirus may lead to secondary bacterial and/or fungal infections which results in high mortality rate among patients, especially in high-risk groups[5,6]. A systematic review showed that bacterial co-infection was relatively infrequent in hospitalized patients with COVID-19[7]. However, the occurrence and characterations of bacterial infection in COVID-19 patients remains poorly understood. 
The bacterial communities of the upper respiratory tract (URT) can prevent respiratory pathogens from establishing an infection on the mucosal surface and spread to the lower respiratory tract (LRT)[8] and play an important role in human health. Concerns about biomarkers of the gut and respiratory microbiome in COVID-19 patients have been raised. For instance, compared with healthy controls, a relative higher abundance of opportunistic pathogens, such as Streptococcus, Rothia, Veillonella and Actinomyces was observed in COVID-19 patients[9]. Moreover, according to previous studies, viral load[10], acute severity[11,12], host immunity and pathogen susceptibility[13] were influenced by the viralbacterial relationships. However, little is known about the interaction between respiratory tract (RT) microbiome and COVID-19 severity, and no appropriate biomarkers are available to support the prognosis of the disease. A cross-sectional study was conducted to explore the relationship between RT microbiome and COVID-19 severity, which could provide a theoretical reference for clinical diagnosis and intervention.

\section{Materials And Methods}

\section{Study design and specimenscollection}

To investigate the characteristics of the lung microbiome in COVID-19 inpatients with different disease severity and explore the microbial biomarkers, we designed a retrospective case-control study.From March 17, 2020 to March 28, 2020, sixty-nine RT samples were collected from 69 patients in a COVID-19 treatment hospital in Wuhan, China. These patients were diagnosed as confirmed COVID-19 cases according to the Diagnosis and Treatment Protocol for Novel Coronavirus Pneumonia(Trial Version 7) released by National Health Commission \& State Administration of Traditional Chinese Medicine[14] .

If serial samples were collected from the same patient, only the first sample was included in the comparison of the microbiome in hospitalized COVID-19 patients with different disease severity. A total of 69 cases were classified as moderate cases, severe cases and critical cases. Finally, 41 moderate cases were classified as mild group, 26 severe cases and 2 critical cases were combined into severe group for the further study.

64 nasopharyngeal swabs, 5 sputum samples were collected at admission from hospitalized COVID-19 patients and stored at $-80^{\circ} \mathrm{C}$ until analysis after collection. Moreover, all blood samples from all patients were stored at room temperature and then were used to analyze routine blood parameters and inflammatory factors by the conventional laboratory methods. All detailed information of clinical characteristics for patients were presented in Supplementary Table S1.

\section{DNA extraction}

Total microbial DNA was extracted from all samples using respiratory tract gene detection kit (Capital Bio Corporation) following the manufacturer's protocol. A beat-beating step was used to lyse bacteria, so that nucleic acids were released into the solution of nucleic acid extraction, and finally the purpose of extracting nucleic acids was achieved. When extracting nucleic acids from sputum samples, it is important to transfer each sputum sample to a sterile centrifuge tube and then add equal volume of $10 \%$ 
$\mathrm{NaOH}$, respectively. Next, these tubes containing sputum samples were vibrated by pulse-vortexing and then liquefied for 30 minutes at $37^{\circ} \mathrm{C}$, in order to achieve better effect in the following operation. When the liquefied sputum samples with no sense of stickiness and homogenization were ideal. Then taking the $1 \mathrm{ml}$ liquefied from each sample into a $1.5 \mathrm{ml}$ centrifuge tube, centrifuging for $5 \mathrm{~min}$ at $12000 \mathrm{rpm}$, and then discarding the supernatant. Adding $1 \mathrm{ml}$ washing solution to the centrifugal tube and vortexing oscillation, centrifuging for $5 \mathrm{~min}$ at $12000 \mathrm{rpm}$ and discarding the supernatant. Next, after adding $100 \mu \mathrm{l}$ nucleic acid extraction solution to the centrifugal tube, the tube was placed in the rapid nucleic acid extractor and oscillated 5 minutes with the maximum vibration velocity. Finally, putting these tubes in a $95^{\circ} \mathrm{C}$ dry bath for heating 5 minutes and then centrifuging 3 minutes at $10000 \mathrm{rpm}$. All samples were stored at $-80^{\circ} \mathrm{C}$ until shipment to Beijing Genomics Institute (BGI) for sequencing.

\section{SrRNA gene sequencing and bioinformatics analysis}

After PCR amplification targeting 16S ribosomal RNA sequencing of the V3-V4 regions in an Illumina MiSeq platform with base-pair reads, DNA libraries was generated. The samples were amplified using the 341F (ACTCCTACGGGAGGCAGCAG) and 806R (GGACTACHVGGGTWTCTAAT) primer pair and sequenced as previously described.

The raw sequencing reads were removed with primers and adapter contaminations using cutadapt v2.6 [15]and quality-filtered to obtain clean data. The quality-filtered reads were denoised and generated Amplicon Sequence Variants (ASVs) by DADA2 (Divisive Amplicon Denoising Algorithm) in Qiime2 (https://qiime2.org/)[16]. Then, ASVs representative sequences were taxonomically classified using Ribosomal Database Project (RDP) Classifier (1.9.1) (http://rdp.cme.msu.edu/)[17]with a minimum confidence threshold of 0.6 against the 16S rRNA database (RDP: Release16 20160930)[18].

Alpha diversity and beta diversity were estimated and rare faction curve was plotted by Microbiome Analyst[19,20]at the ASVs level, respectively. Alpha diversity was estimated by the Simpson index and ACE index. Beta diversity analysis was performed by principal coordinate analysis (PCOA) of the ordination method based on the Bray-Curits index. For the important biomarkers identification, a two-step method was adopted by MicrobiomeAnalyst (https://www.microbiomeanalyst.ca/). First step, a random forest model (ntree $=500$ ) was used to estimate the difference between the two groups and top 10 genera were selected as candidate biomarkers by their contributions to classification accuracy (MDA, Mean Decrease Accuracy). Second step, the linear discriminant analysis effect size (LEfSe) method was used to distinguish between the mild group and the severe group and genera with LDAscore $>4$ [9] were selected as candidate biomarkers. Finally, the common genera were identified as final biomarkers by the two-step method.

\section{Statistical Analysis}

All the statistical analyses were performed with $R$ statistical software packages ( $R$ version 3.4.1). As appropriate, comparisons between different rates or percentages were performed using the Chi-square test or the Fisher's Exact test. Continuous variables such as age, inpatient days and clinical laboratory 
test results were reported as median and interquartile interval (IQR), and statistical comparisons by the Mann-Whitney U test. It was supposed to be the Point-Biserial Correlation Coefficient, when we explored the relationships between partial clinical indexes as binomial variables and the top 10 genera. But We assigned these indexes as dummy variables and then explored the relationships directly through the spearman's rank test. A Pvalueof less than 0.05 was considered as statistically significant.

\section{Results}

\section{Demographic and clinical characterizations of patients}

Demographic information and clinical characteristics of 69 COVID-19 patients were shown in Table 1 and laboratory test indices between two groups were shown in Table 2. The median age of patients was 57 years (IQR:49.5-70.5), the median of length of hospitalization was 12 days (IQR:6.5-17.0) and 14 (34.1\%) were men in the mild group. In the mild group, $73.2 \%$ of hospitalized patients had at least one underlying disease, and $4.9 \%$ were admitted into the ICU. In the severe group, the median age was 71 years (IQR:62.382.8) and the median duration of hospitalization was 18.5 days (IQR:10.0-38.6) and 16 were men $(57.1 \%)$. And there was $92.9 \%$ of the patients with underlying diseases and $32.1 \%$ of patients were admitted into the ICU.

There were significant differences in age, inpatient days, dyspnea, coexisting disorders, ICUadmission $(p<0.05)$ between the mild group and the severe group $(p<0.05)$. Significant differences were also found in white blood cell count (WBC), neutrophil count (GRA), lymphocyte count (LYMPH), C-reactive protein (CRP), and procalcitonin between two groups $(p<0.05)$. However, there was missing data of procalcitonin in 8 patients and it was not considered in the subsequent analysis.

\section{Table1 Demographic and clinical characteristics between two patient groups}




\begin{tabular}{|llll|}
\hline & \multicolumn{2}{l}{ Groups } & \\
\cline { 2 - 3 } Characteristics & Mild group $(\mathrm{n}=41)$ & Severe group(n=28) & P-value \\
\hline Age[median(IQR)] & $57.0(49.5-70.5)$ & $71.0(62.3-82.8)$ & 0.009 \\
\hline BMI(kg/m²) & $25.0(20.3-26.0)$ & $24.0(20.5-27.2)$ & 0.869 \\
\hline Inpatient days & $12.0(6.5-17.0)$ & $18.5(10.0-38.6)$ & 0.010 \\
\hline Sex,male(\%) & $14(34.1)$ & $16(57.1)$ & 0.058 \\
\hline Symptoms(yes,\%) & & & \\
\hline Cough & $31(75.6)$ & $25(89.3)$ & 0.154 \\
\hline Diarrhea & $7(17.1)$ & $4(14.3)$ & 1.000 \\
\hline Dyspnea & $4(9.8)$ & $11(39.3)$ & 0.003 \\
\hline Coexisting disorders(\%) & & & 0.040 \\
\hline Any & $30(73.2)$ & $26(92.9)$ & 0.329 \\
\hline Hypertension & $20(48.8)$ & $17(60.7)$ & 0.256 \\
\hline Cardiopathy & $7(17.1)$ & $8(28.6)$ & 0.007 \\
\hline ICU admission (\%) & $2(4.9)$ & $9(32.1)$ & 0.389 \\
\hline Death during hospitalization(\%) & $2(4.9)$ & $3(10.7)$ & \\
\hline
\end{tabular}

Abbreviation: IQR, interquartile range; BMI, body mass index; ICU, intensive care unit.

${ }^{*}$ P-value $<0.05$ was considered statistically significant between the mild group and severe group.

Table2 Laboratory test results between two patient groups 


\begin{tabular}{|c|c|c|c|}
\hline & \multicolumn{2}{|l|}{ Groups } & \multirow[b]{2}{*}{$P$-value ${ }^{\star \star}$} \\
\hline & Mild group $(n=41)$ & Severe group $(n=28)$ & \\
\hline $\operatorname{lgG}[\mathrm{ug} / \mathrm{mL}$,median(IQR)] & $121.3(58.3-187.6)$ & $102.8(55.6-188.1)$ & 0.903 \\
\hline IgM[ug/mL,median(IQR)] & $16.3(3.7-33.5)$ & $18.0(5.8-39.0)$ & 0.961 \\
\hline White blood cell count, $\left(\times 10^{9} / \mathrm{L}\right)$ & $6.0(5.2-8.0)$ & 7.6(6.1-10.6) & 0.006 \\
\hline Neutrophil count, $\left(\times 10^{9} / \mathrm{L}\right)$ & $3.9(3.1-4.7)$ & $5.7(4.6-8.7)$ & 0.001 \\
\hline Lymphocyte count, $\left(\times 10^{9} / \mathrm{L}\right)$ & $1.6(1.2-1.9)$ & $0.9(0.5-1.8)$ & 0.006 \\
\hline Hemoglobin $(\mathrm{g} / \mathrm{L})$ & $117.0(100.5-133)$ & 111.5(93.0-133.8) & 0.591 \\
\hline C-reactive protein $(\mathrm{mg} / \mathrm{L})$ & $4.0(1.5-9.6)$ & $42.4(15.3-72.3)$ & $\varangle 0.001$ \\
\hline Procalcitonin (ng/m) & $0.04(0.03-0.17)^{\star}$ & $0.15(0.06-0.38)^{\mathrm{a}}$ & 0.002 \\
\hline
\end{tabular}

Abbreviation:IgG: Immunoglobulin G; IgM:Immunoglobulin M

*Partial data missing; ** P-value $<0.05$ was considered statistically significant between the mild group and severe group.

\section{Microbial richness, abundance and diversity}

Sixty-nine specimens including 64 nasopharyngeal swabs and 5 sputum swabs were analyzed by $16 \mathrm{~s}$ rRNA gene sequencing to study the microbial composition ofrespiratory tract in COVID-19 patients. After merging and filtering the raw reads data, 3957195 high-quality sequence reads were saved for the subsequent analysis. In order to avoid analysis bias caused by different sequence reads of samples, all samples were rarefied to even sequencing depth based on the sample with the lowest sequencing depth. With the increase of sequencing depth, the rarefaction curves increased rapidly and then became flat (Fig. 1), indicating that the sample sequencing data was reasonable and the quality of reads was good with certain depth and representativeness.

According to Simpson diversity index $(P=0.0062)$ and ACE diversity index $(P=1.7474 \mathrm{e}-5)$, the microbial diversity and the richness of mean community were significantly higher in the mild group than the severe group (Fig.2A, B). PCoA based on Bray-Curtis distances displayed differences in both the mild group and severe group (analysis of similarities, ANOSIM, R=0.143, P冈0.003) (Fig. 3).

\section{Different bacterial taxonomic characterizations in the two groups}

To investigate alterations in microbiome of the respiratory about COVID-19 patients with different severity, we selected top ten relative abundances at the phylum, class, order, family and genus levels respectively and assessed differences by Mann-Whitney $U$ test in the groups. Then, relative abundances with significant differences at different taxonomic levels were shown in Figure 4 and Table S3. At the 
phylum level, the relative abundances of Fusobacteria and Bacteroideteswere were lower in the severe group compared with the mild group (Fig. 4A), which was probably due to significant decrease of FusobacteriiaandBacteroidiaat the class level (Fig. 4B). At the class level, except the significant reduction

in Bacteroidia and Fusobacteriia, the relative abundances of Clostridia and Negativicutesdecreased in the severe group. At the order level (Fig. 4C), there were seven significant orders including Bacteroidales, Clostridiales, Enterobacteriales, Fusobacteriales, Neisseriales, Pseudomonadales, Selenomonadales, among which the relative abundances of Selenomonadales and Bacteroidales in the mild group were higher than that in the severe group. However, the relative abundance of Pseudomonadales was lower in the mild group than that in the severe group. At the family level(Fig. 4D), the relative abundances of Actinomycetaceae, Micrococcaceae, Prevotellaceae, Streptococcaceae, Veillonellaceaesignificantly decreased in the severe group compared to the mild group. At the genus level,the relative abundances of Actinomyces, Prevotella, Rothia, Streptococcus, Veillonelladecreased in the severe group (Fig. 4E) and this taxonomy bar plot showed the main species composition of each sample (Fig. 5, Table S2), respectively

\section{Biomarkers exploration in the different groups}

The random forest model was used to distinguish the different genera of respiratory microbiome between the two groups. The differential genera were ranked by their contribution to the mild and severe disease groups and then the top ten genera were selected as candidate biomarkers (Fig. 6A).

LDA-score $₫ 4$ by LEfSe analysis was conducted to select candidate biomarkers in the groups. Significant differences were found in twenty-two genera of Acinetobacter, Capnocytophaga, Centipeda, etc., between the mild group and severe group (Fig. 6B). Finally, eighty common genera

(Treponema,Lachnoanaerobaculum, Parvimonas, Selenomonas, Alloprevotella, Porphyromonas, GemellaandStreptococcus) were selected to distinguish the two groups. The correlations of enriched genera were analyzed by Spearman's rank test to evaluate relationships among genera (Fig. 6C). there were positive correlations across different genera with different degrees of COVID-19 severity, except for Acinetobacter.

\section{Relationship between respiratory microbiome composition and clinical characteristics}

Spearman's rank analysis was used to evaluate the relationships of correlation between top 10 genera and clinical characteristics (including age, inpatient days, dyspnea, coexiting diseases and days in the ICU) and indexes (WBC, GRA, LYMPH and CRP) in the mild group and severe group, respectively (Fig. 7, Table S4). According to Figure 7A and 7B, the genera of Actinomyces and Prevotella were negatively correlated with age in the two groups. The genera of Acinetobacter and Streptococcus were positively correlated with dyspnea in the severe group, but these genera were uncorrelated with dyspnea in the mild group. Moreover, inpatient days, ICU admission, GRA and LYMPH were correlated with different genera in the two groups. In addition, there were a positive correlation between Klebsiella and WBC in two groups and a negative correlation between Rothiaand WBC in the mild group. Acinetobacter, Actinomyces, Corynebacterium, Rothia and Streptococcus were negatively correlated with CRP in the severe group, but there was no correlation between top 10 genera and CRP in the mild group (Fig. 7C, D). 


\section{Discussion}

In this cross-sectional study, we explored connections between COVID-19 inpatients with different severity and respiratory tract microbiome. While a large number of studies focused on SARS-CoV-2, there were few studies investigating alterations of the microbiome of respiratory tract between the mild and severe groups. Upper respiratory tract is the entry point of respiratory viruses and bacterial pathogens[8]. The initial viral replication occurs in the nasal epithelia of COVID-19 patients[21] and when this initial response is insufficient, the virus migrates into the LRT and then results in moderate or severe COVID19[21]. Therefore, there is a need to understand the alterations of respiratory tract microbiome during SARS-CoV-2 infection.

In this study, we characterized the alterations of respiratory tract microbiome in COVID-19 inpatients with different severity. Our results showed that there were significant differences between mild and severe groups, which is in accordance with previous studies[11,12]. Although these studies had various group reference standards, they all showed that microbiome diversity and abundance correlated inversely with disease severity of COVID-19 inpatients.

Compared with the mild group, our study found there was a decreased relative abundance of opportunistic pathogens, including Actinomyces, Prevotella, Rothia, Streptococcus, Veillonella, in severe COVID-19 patients. The relative abundance of Actinomyces, Rothia and Streptococcus were negatively associated with CRP. Rothia was thought to play an important role in the pathogenesis of pneumonia, especially for patients with retained catheters and immunocompromised patients[22]. A study suggested that Rothia and Streptococcus were connected with susceptibility to secondary bacterial lung infection in patients of avian H7N9 virus infection[23]. Noteworthily, the level of Rothia might also affect the pathogenesis or disease severity of SARS-CoV-2 infection, and the changes of Rothia and Streptococcus might be associated with with susceptibility to secondary bacterial lung infection in patients of COVID-19, while further explorations are needed.

Several bacterial taxa in respiratory tract microbiome have been found to be associated with disease severity in COVID-19 inpatients and can become potential biomarkers to predict the development of disease, which was similar with previous studies[23]. Although we combined the random forest model and LEfSe to find these potential biomarkers, the final results were both partially overlapping and different. Therefore, it is necessary to find a more appropriate method to identify the microbial biomarkers. Most of our samples were nasopharyngeal swabs, which lead to final biomarkers associated with oral related bacteria. Thus, we hypothesized that the oral cavity may be a potential source of the pathogens that infect the lung. A study hypothesized that the genera of oral bacteria enriched in COVID19 such as Veillonella and Megasphaera may be pathogenic when transferred to other organs of the body[24], which is in accordance with our hypothesis.

Another important finding was a coresymbiotic relationships between most enriched genera in COVID-19 inpatients with different disease severity, except there was the negative correlation between Acinetobacter and other enriched genera. More than one study about coinfection with Acinetobacter 
baumanniisecondary to SARS-CoV-2 infections, particularly in ICU, during the COVID-19 pandemic has been reported[25], which suggested that there were nosocomial infections. Moreover, bacterial copathogens are not only commonly identified in viral respiratory infections, but also important causes of exacerbating condition.

Our study had several limitations. Firstly, this study is a cross-sectional study which makes it impossible to figure out whether the infection of SARS-CoV-2 affects the respiratory community or whether the dominated bacteria types of respiratory tract community select a person to viral infection. This is a complex relationship. Secondly, there are some unknown classification taxa at the species level due to limitation of the $16 \mathrm{~s}$ rRNA sequencing. Thirdly, there are still some confounding factors such as antibiotic use, age and sex impacting the characteristics of respiratory tract microbiome in COVID-19 inpatients. Though compared to the influence of SARS-CoV-2 infection, age and sex might have a limited effect on the overall composition of the respiratory tract microbiome. Thus, future studies should control or match these confounding factors. Lastly, our results should be extended cautiously, because our study only have less than 100 samples (64 nasopharyngeal swabs and 5 sputum specimens) and cannot represent all population. In spite of these limitations, our study shed some light in the interactions between characteristics of respiratory bacterial microbiome and SARS-CoV-2 in COVID-19 patients with different disease severity.

\section{Conclusion}

In this study we have demonstrated that COVID-19 disease severity can be predicted by respiratory tract microbiome compositions with high performance. Particularly, eight potential biomarkers can serve as indicator species to robustly predict the severity of SARS-CoV-2 infections. Our discovery about bacterial communities in COVID-19 with different severity suggests that bacterial microbiome might play an important role in clinical diagnosis.

\section{Abbreviations}

COVID-19: Coronavirus disease 2019; rRNA: 16S ribosomal RNA; ICU: Intensive Care Unit; GRA: neutrophil count; LYMPH: lymphocyte count; WBC: white blood cell count; CRP: C-reactive protein; SARS-CoV-2: syndrome coronavirus 2; RT: respiratory tract; URT: the upper respiratory tract; LRT: the lower respiratory tract; BGI: Beijing Genomics Institute; ASVs: Amplicon Sequence Variants; DADA2: Divisive Amplicon Denoising Algorithm; RDP: Ribosomal Database Project; PCoA: principal coordinate analysis; MDA: Mean Decrease Accuracy; LEfSe: linear discriminant analysis effect size; IQR: interquartile interval; BMI: body mass index; ANOSIM: analysis of similarities.

\section{Declarations}

\section{Acknowledgements}


We are grateful to all contributor, including these researchers and these patients during the study.

\section{Authors'contributions}

$\mathrm{CW}$ and $\mathrm{YC}$ designed the research. $\mathrm{WL}, \mathrm{CY}, \mathrm{RJ}, \mathrm{YK}, \mathrm{LJ}$, and $\mathrm{XL}$ collected the field samples. $\mathrm{LJ}$ performed the DNA isolation. JC, YC, CW, XLandJG conducted formal analysis, interpreted results, and wrote the manuscript. All authors read and approved the final manuscript.

\section{Funding}

The study was supported by a grant from National key research and development program (2019YFC1200501), National Natural Science Foundation of China (12171295), Beijing Nova Program (Z181100006218107).

\section{Availability of data and materials}

The human metagenomics sequencing data during this study are available in the in the NCBls sequence read archive (SRA) database and the NCBI BioSample database repository. The BioProject ID is PRJNA780081.

\section{Ethics approval and consent to participate}

The study protocol was approved by the Ethics Committee of Chinese PLA Center for Disease Control and Prevention, and the requirement for informed consent was waived by the Ethics Committee.

\section{Consent for publication}

Not applicable.

\section{Competing interests}

The authors declare that they have no competing interests.

\section{References}

1. Chan JF-W, Yuan S, Kok K-H, et al. A familial cluster of pneumonia associated with the 2019 novel coronavirus indicating person-to-person transmission: a study of a family cluster. Lancet Lond Engl. 2020;395:514-23.

2. World Health Organization. WHO Coronavirus (COVID-19) Dashboard: Global situation [Internet]. 2021 [cited 2021 Sep 13]. Available from: https://covid19.who.int/

3. Cucinotta D, Vanelli M. WHO Declares COVID-19 a Pandemic. Acta Bio-Medica AteneiParm. 2020;91:157-60.

4. de Wit E, van Doremalen N, Falzarano D, et al. SARS and MERS: recent insights into emerging coronaviruses. Nat Rev Microbiol. 2016;14:523-34. 
5. Bakaletz LO. Viral-bacterial co-infections in the respiratory tract. CurrOpin Microbiol. 2017;35:30-5.

6. Zhou F, Yu T, Du R, et al. Clinical course and risk factors for mortality of adult inpatients with COVID19 in Wuhan, China: a retrospective cohort study. Lancet Lond Engl. 2020;395:1054-62.

7. Langford BJ, So M, Raybardhan S, et al. Bacterial co-infection and secondary infection in patients with COVID-19: a living rapid review and meta-analysis. Clin Microbiol Infect Off Publ Eur Soc Clin Microbiol Infect Dis. 2020;26:1622-9.

8. Man WH, de SteenhuijsenPiters WAA, Bogaert D. The microbiota of the respiratory tract: gatekeeper to respiratory health. Nat Rev Microbiol. 2017;15:259-70.

9. Gu S, Chen Y, Wu Z, et al. Alterations of the Gut Microbiota in Patients With Coronavirus Disease 2019 or H1N1 Influenza. Clin Infect Dis Off Publ Infect Dis Soc Am. 2020;71:2669-78.

10. Rosas-Salazar C, Kimura KS, Shilts MH, et al. SARS-CoV-2 infection and viral load are associated with the upper respiratory tract microbiome. J Allergy Clin Immunol. 2021;147:1226-1233.e2.

11. Merenstein C, Liang G, Whiteside SA,et al. Signatures of COVID-19 Severity and Immune Response in the Respiratory Tract Microbiome. mBio. 2021;12:e0177721.

12. Zhong $\mathrm{H}$, Wang $\mathrm{Y}$, Shi Z,et al. Characterization of respiratory microbial dysbiosis in hospitalized COVID-19 patients. Cell Discov. 2021;7:23.

13. Rashidi A, Ebadi M, Rehman TU, et al. Effect of COVID-19 precautions on the gut microbiota and nosocomial infections. Gut Microbes. 2021;13:1-10.

14. National Health Commission \& State Administration of Traditional Chinese Medicine. Clinical Protocols for the Diagnosis and Treatment of COVID-19 (Trial Version 7). 2020; 1-17.

15. Marcel martin. Cutadapt removes adapter sequences from high-throughput sequencing reads. EMBnet.journal. 2011;17:10-2.

16. Bolyen E, Rideout JR, Dillon MR, et al. Reproducible, interactive, scalable and extensible microbiome data science using QIIME 2. Nat Biotechnol. 2019;37:852-7.

17. Wang Q, Garrity GM, Tiedje JM, et al. Naive Bayesian classifier for rapid assignment of rRNA sequences into the new bacterial taxonomy. Appl Environ Microbiol. 2007;73:5261-7.

18. Cole JR, Wang Q, Fish JA, et al. Ribosomal Database Project: data and tools for high throughput rRNA analysis. Nucleic Acids Res. 2014;42:D633-642.

19. Dhariwal A, Chong J, Habib S, et al. MicrobiomeAnalyst: a web-based tool for comprehensive statistical, visual and meta-analysis of microbiome data. Nucleic Acids Res. 2017;45:W180-8.

20. Chong J, Liu P, Zhou G, et al. Using MicrobiomeAnalyst for comprehensive statistical, functional, and meta-analysis of microbiome data. Nat Protoc. 2020;15:799-821.

21. Murgolo N, Therien AG, Howell B, et al. SARS-CoV-2 tropism, entry, replication, and propagation: Considerations for drug discovery and development. PLoSPathog. 2021;17:e1009225.

22. Ramanan P, Barreto JN, Osmon DR, et al. Rothia bacteremia: a 10-year experience at Mayo Clinic, Rochester, Minnesota. J Clin Microbiol. 2014;52:3184-9. 
23. Lu H-F, Li A, Zhang T, et al. Disordered oropharyngeal microbial communities in H7N9 patients with or without secondary bacterial lung infection. Emerg Microbes Infect. 2017;6:e112.

24. Ma S, Zhang F, Zhou F, et al. Metagenomic analysis reveals oropharyngeal microbiota alterations in patients with COVID-19. Signal Transduct Target Ther. 2021;6:191.

25. Rangel K, Chagas TPG, De-Simone SG. Acinetobacter baumannii Infections in Times of COVID-19 Pandemic. Pathog Basel Switz. 2021;10:1006.

\section{Figures}

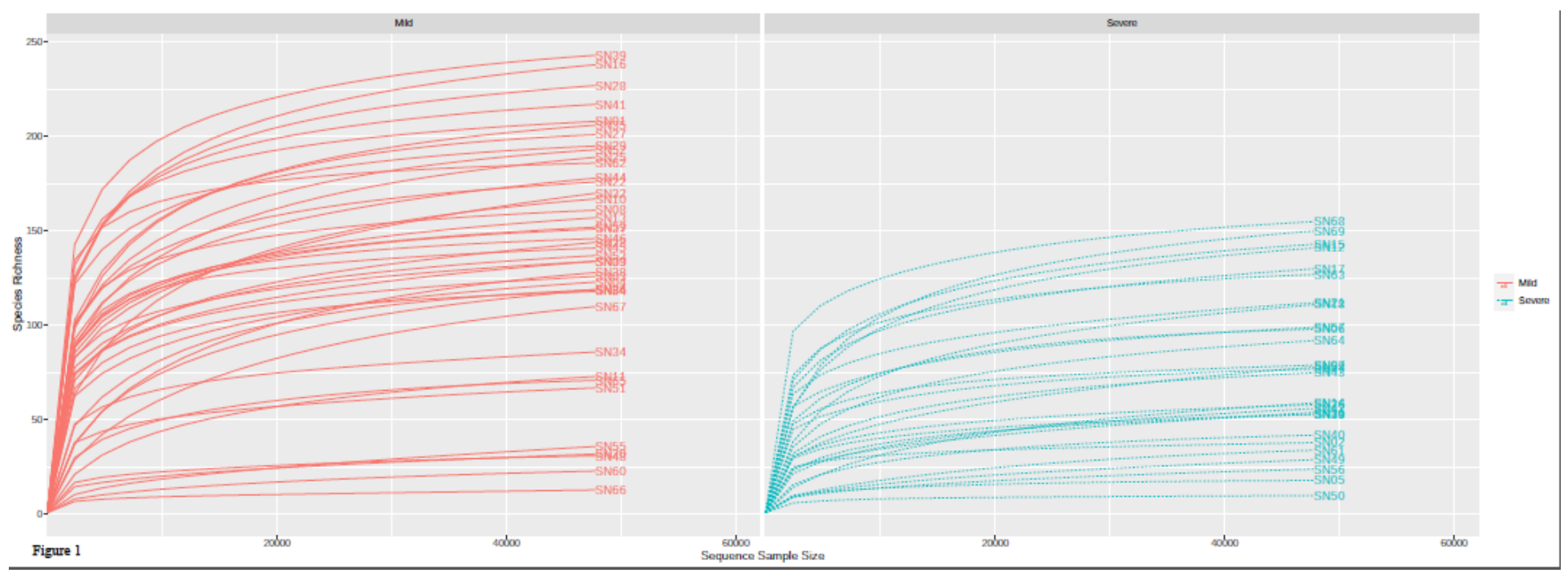

\section{Figure 1}

The rarefaction curves between the mild group and severe group. A curve represents a sample. The $x$-axis indicates the number of clean reads randomly selected from a sample, and the $y$-axis represents the species richness. Words "SN" plus a number indicates a specific sample. 

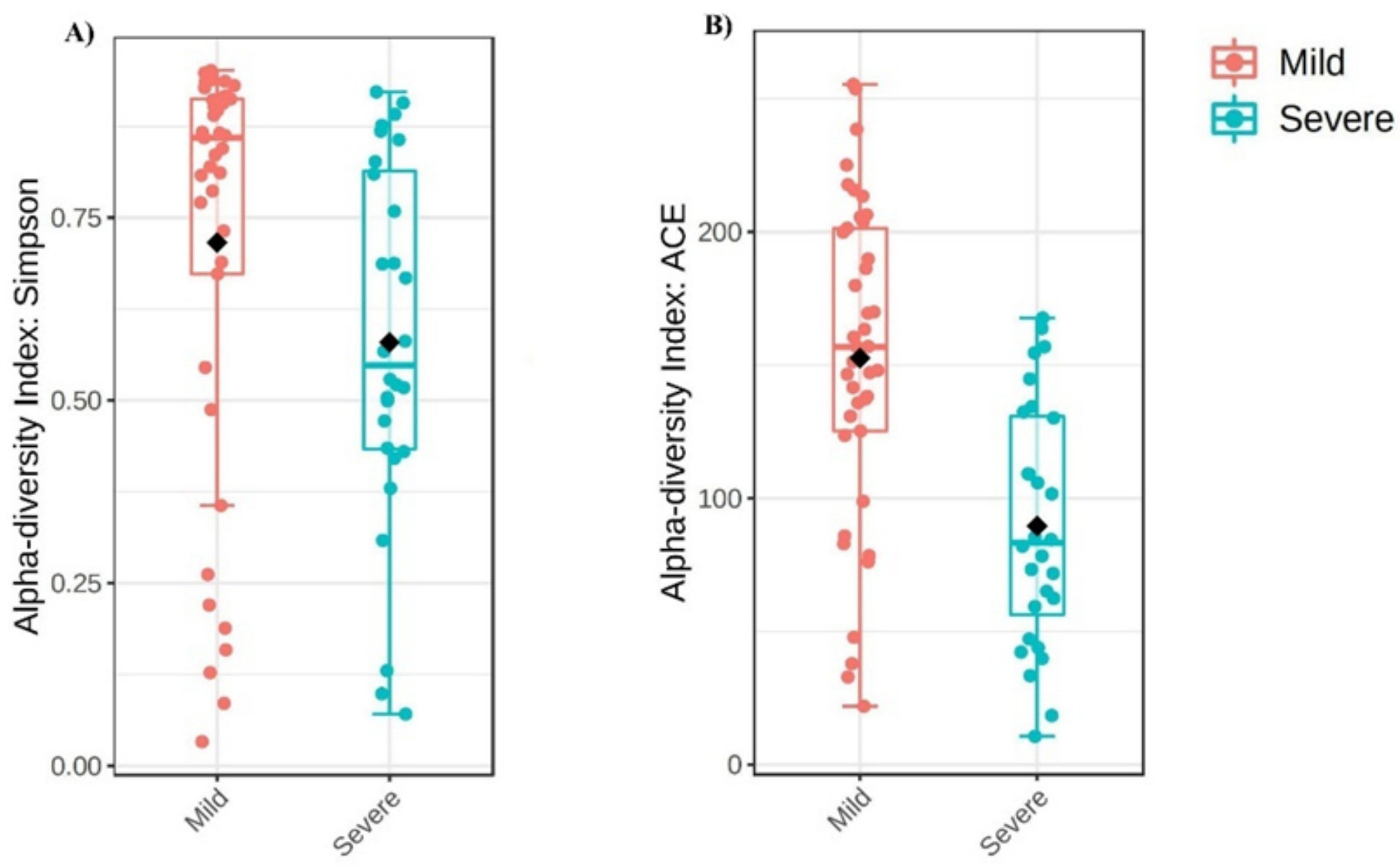

\section{Figure 2}

Alpha diversity between mild group and severe group. The Mann-Whitney $U$ test was used to compare the Simpson index $(p=0.0062)$ and $\operatorname{ACE}(p=1.7474 e-5)$ index between the two groups, respectively. There were significant differences about the upper respiratory microbiome in the groups. 


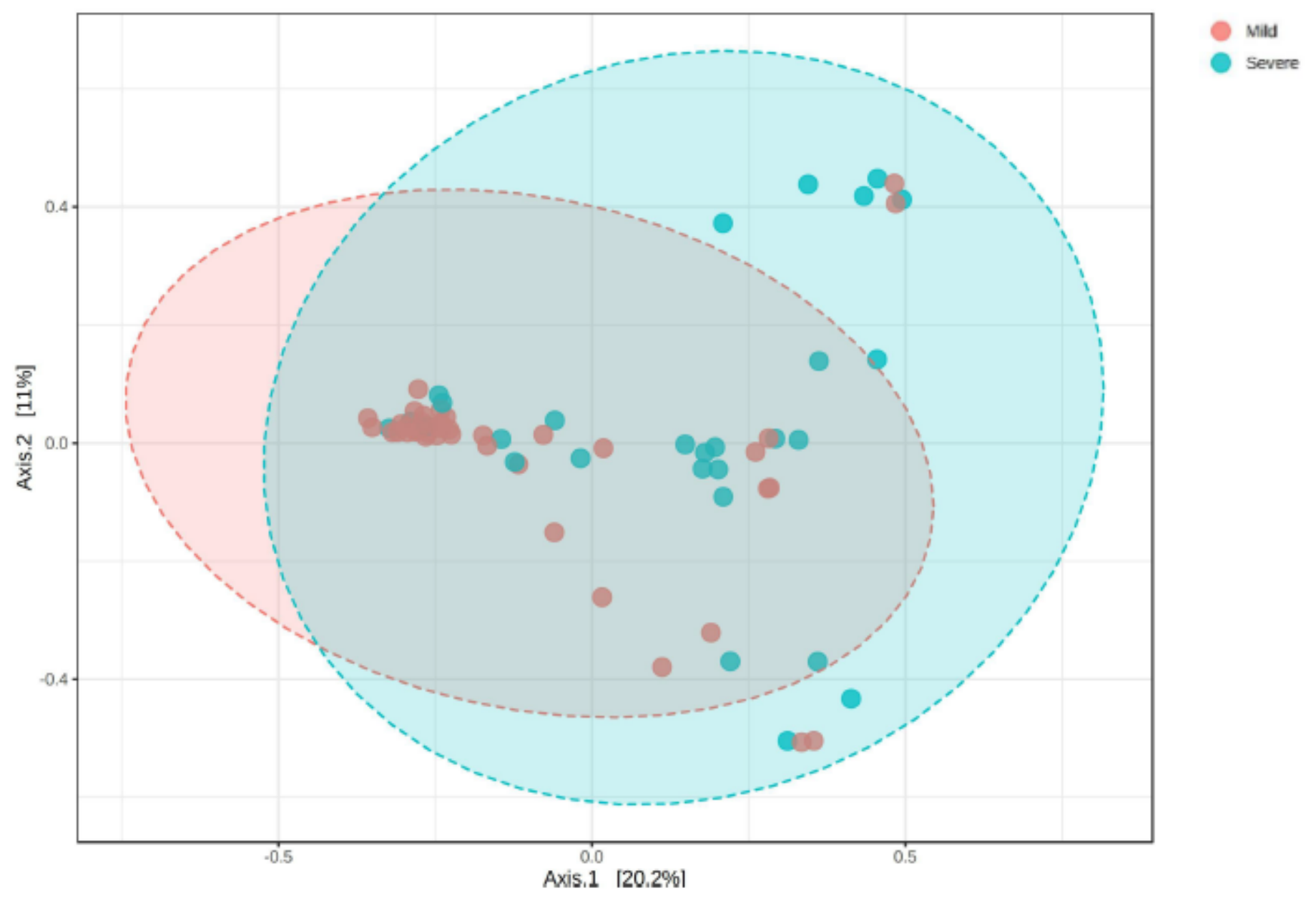

\section{Figure 3}

Beta diversity between mild group and severe group. PCoA of Bray-Curtis distances indicated differences in the mild group (orange) and severe group (green). Each point represents one sample and each color shows all samples from the same group. $\mathrm{R}=0.143, P \otimes 0.003$. 
A)

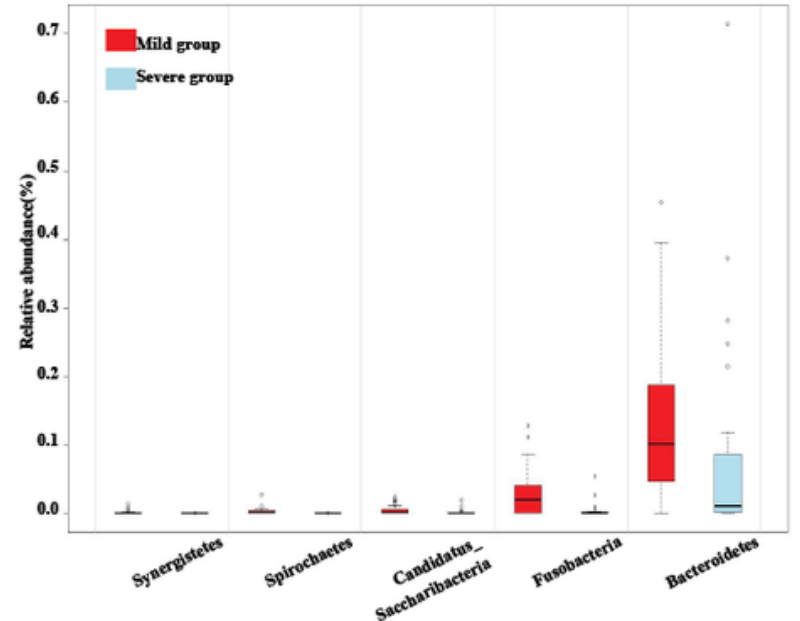

C)

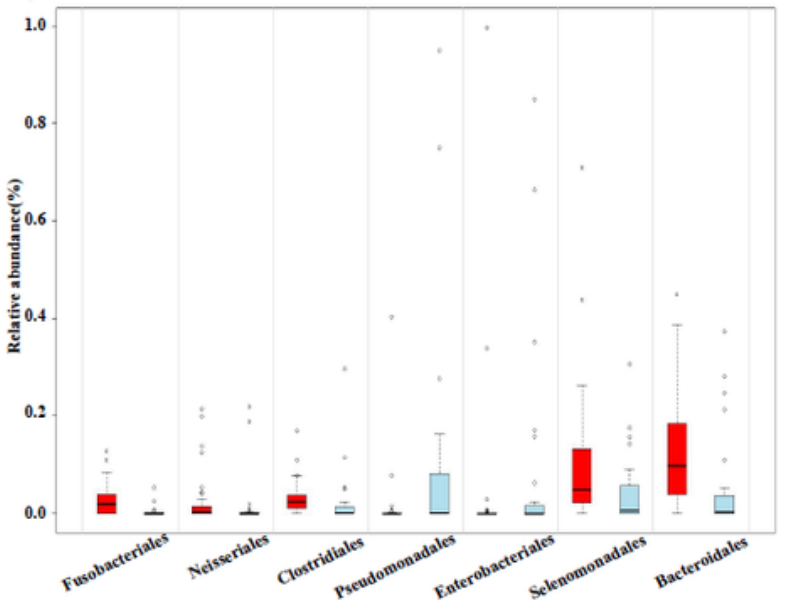

E)

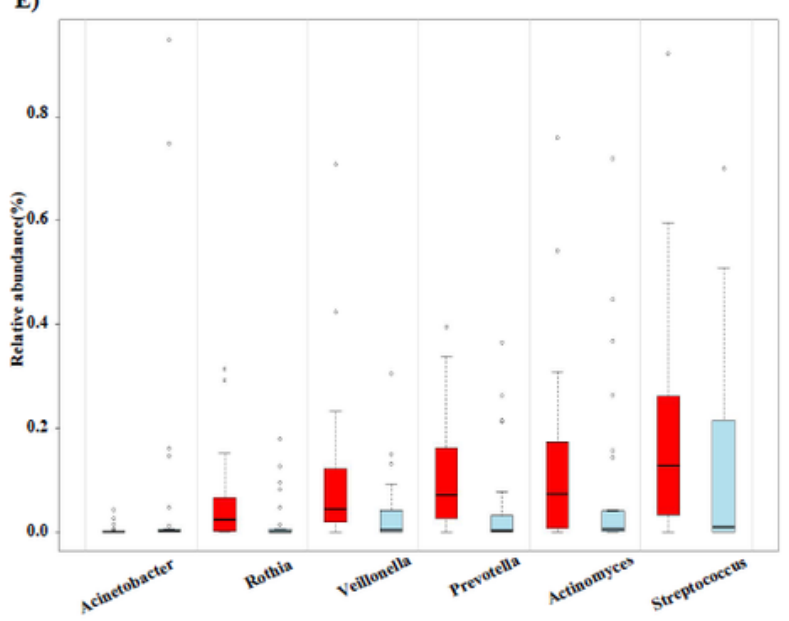

B)

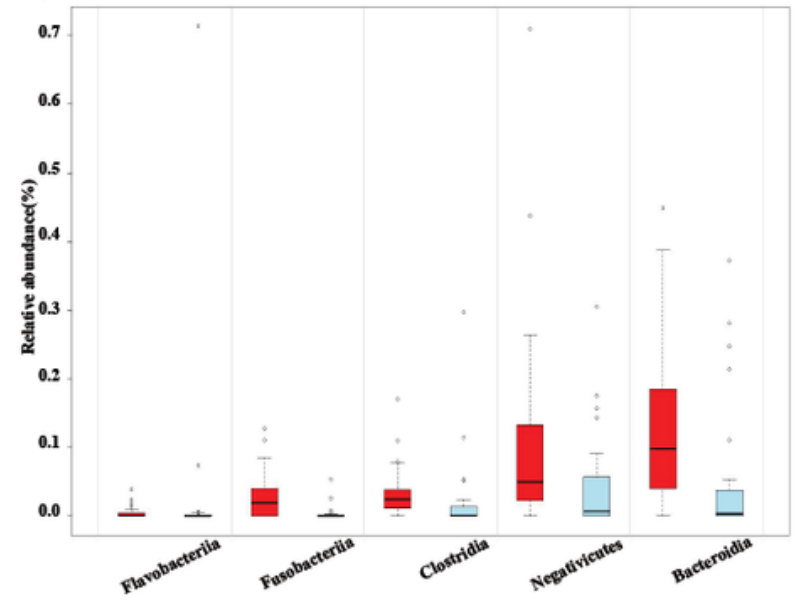

D)

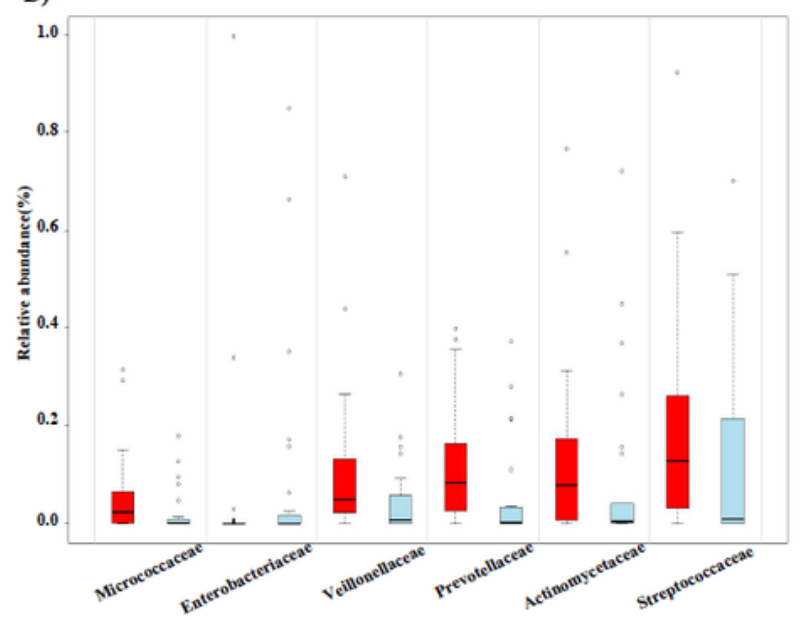

\section{Figure 4}

Taxonomic differences of top 10 in the respiratory microbiome beween the mild group and the severe group. Comparison of the significant relative abundance at the Phylum (A), Class (B), Order (C), Family (D), Genus (E) levels in the groups. Each box shows the relative abundance levels of respiratory microbes in the mild group (red) and the severe group (blue) and the black line represents median abundance. Each 
hollow circle represents these extreme values. All significant taxa were selected by Mann-Whitney $U$ test $(P \otimes 0.05)$.

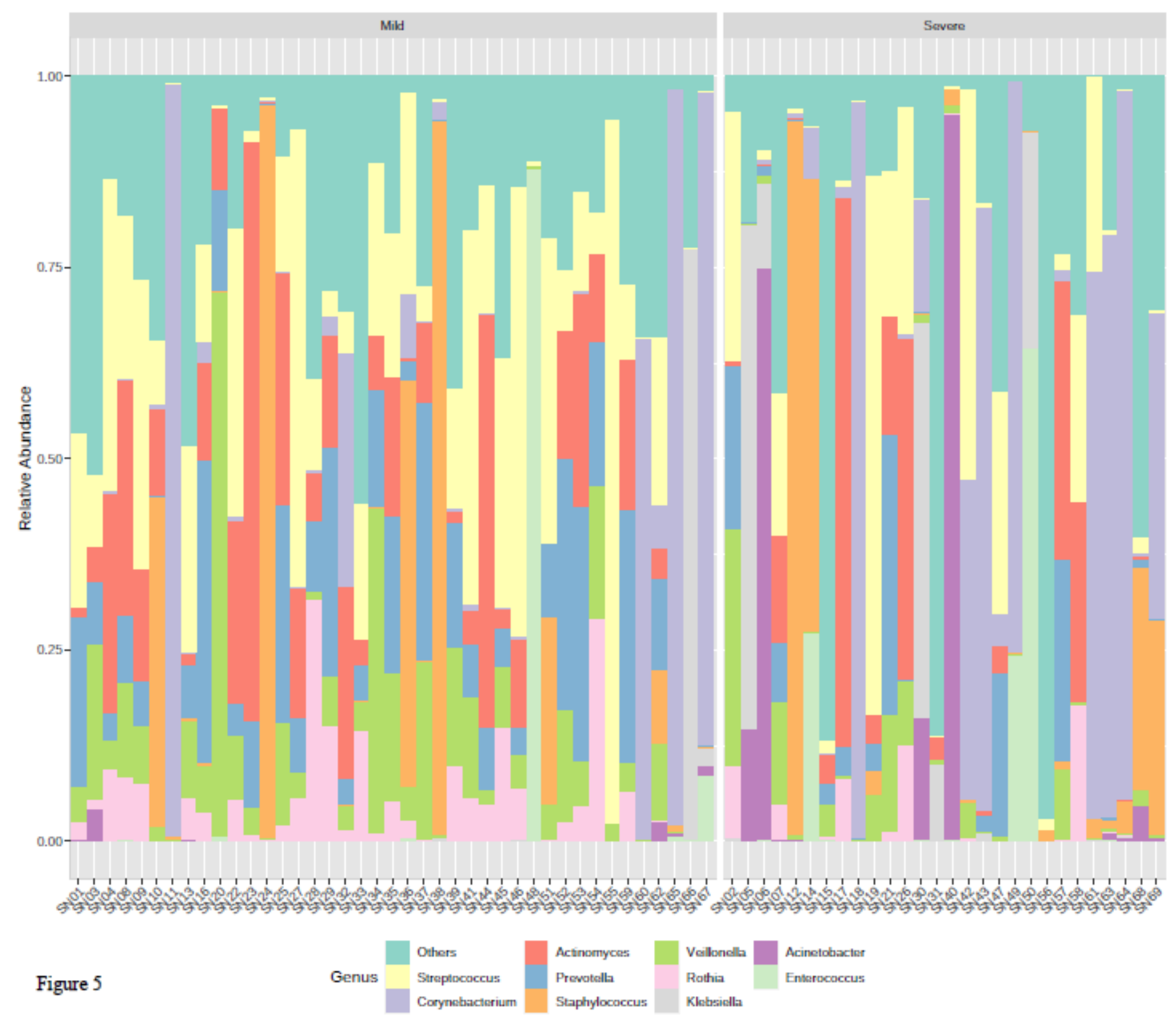

\section{Figure 5}

Taxonomy bar plot showing the top10 microbiota profile at phylum level. This bar plot shows the main species composition of each sample in the two groups at the genus level, respectively. 


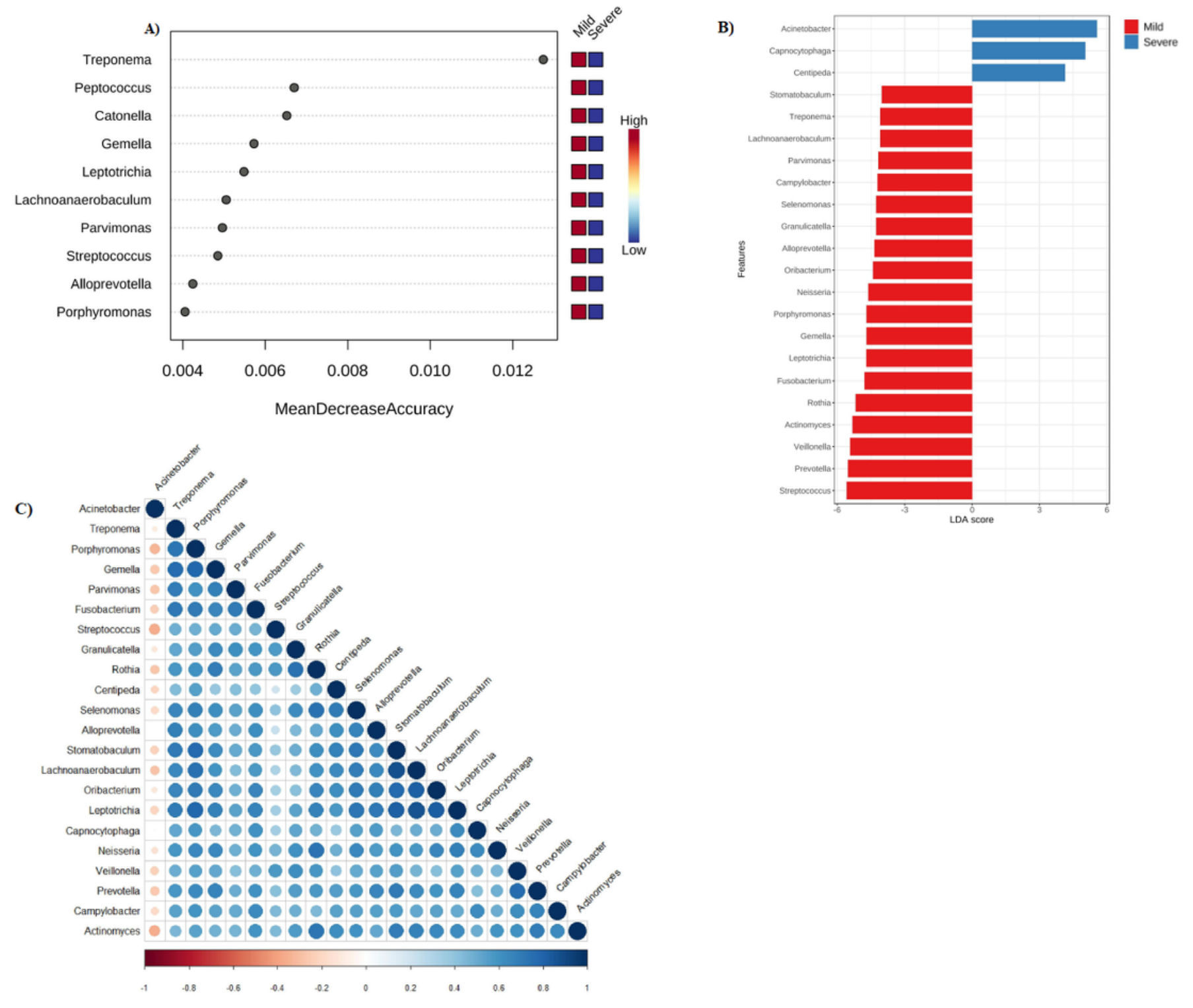

Figure 6

Screening of potential biomarkers and exploration of their correlations. The x-axis represents Mean Decrease Accuracy and $y$-axis indicates the genera; the greater mean decrease accuracy, the more important the genus(A).At the genus level, LDA-scores generated by the Linear discrimination analysis (LDA) effect size (LEfSe) analysis showed significant differences in the mild (red) and severe (blue) groups. LDA-score threshold $₫ 4$ (B). Spearman's rank correlation of different genera between the mild and severe group (C).Blue represents positive correlation and red represents negative correlation. The larger the circle and the deeper the colour, the greater the correlation coefficient in genera. 
A)

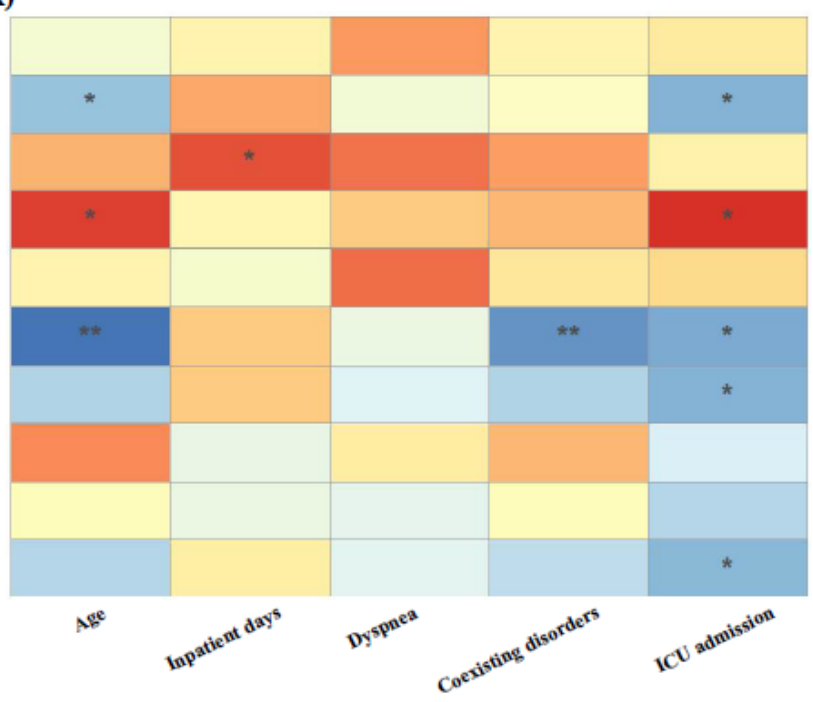

C)

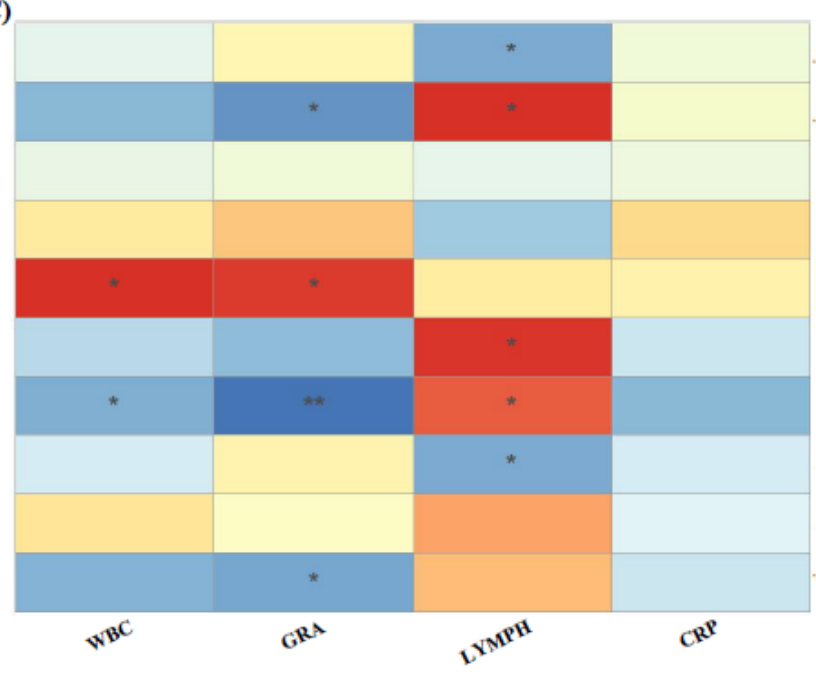

B)

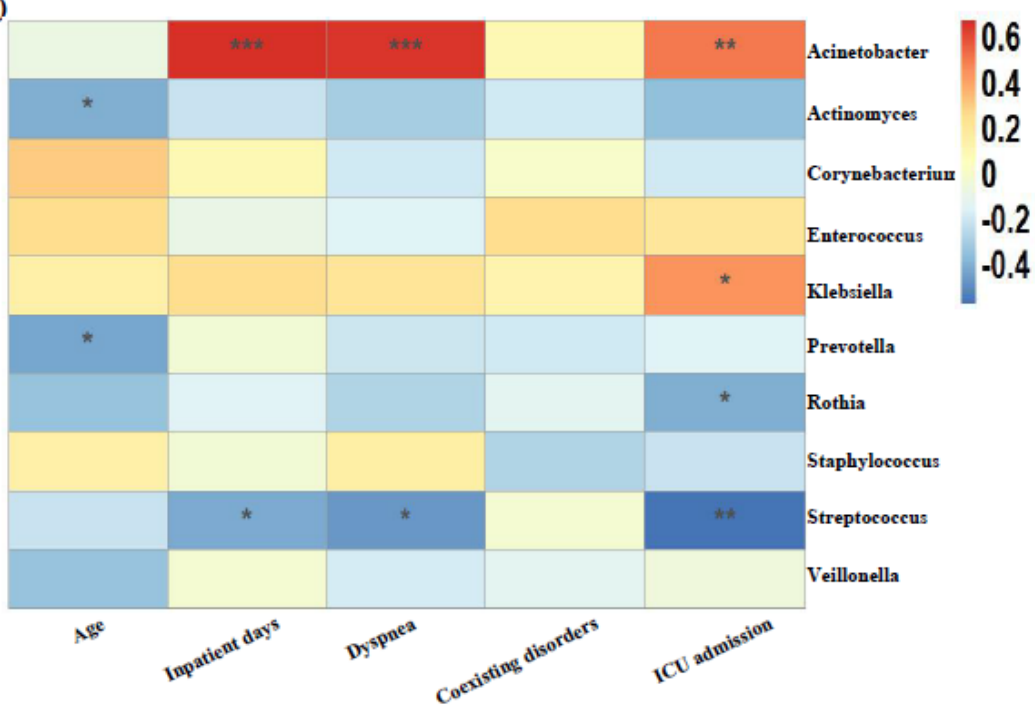

D)

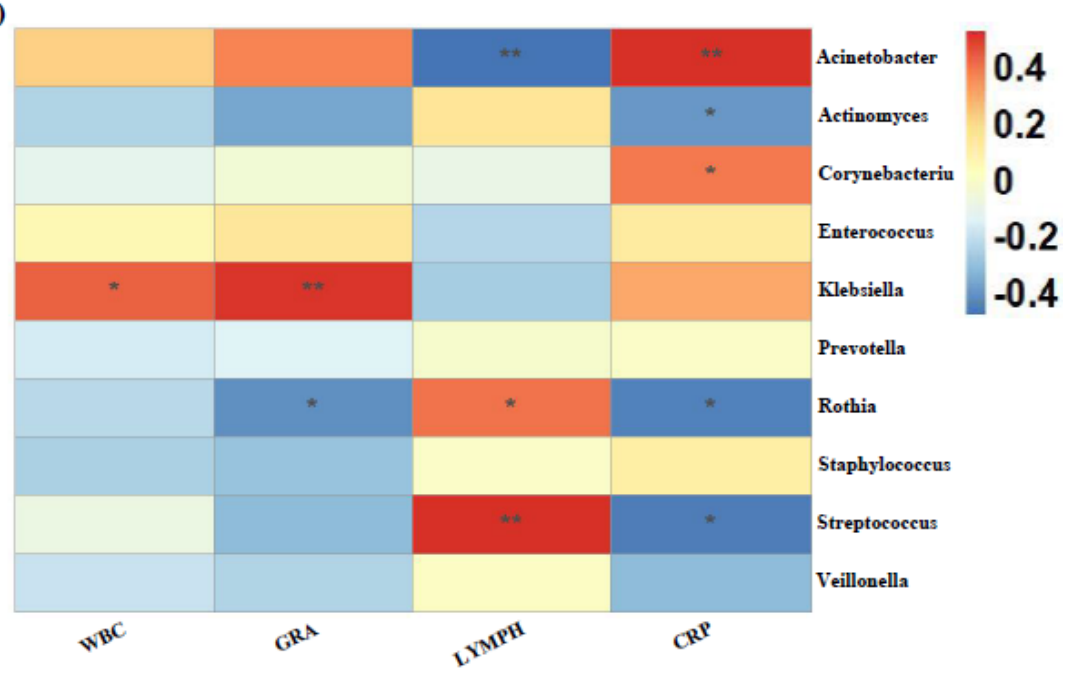

Figure 7

Association between clinical characteristics and genera (Top 10 genera in terms of the relative abundance). Spearman analysis was used to evaluate the correlation of genera and clinical indices in the mild group (A)and the severe group (B). And these laboratory test indices with significant differences in the mild group (C) and the severe group (D), respectively. Clinical variables include age, inpatient days, in the ICU and dyspnea) and laboratory biomarkers include WBC, GRA, LYMPH and CRP. A color gradient from from blue (negative correlation) to red (positive correlation) indicates the degree of correlation. * $P=0.05 \sim 0.01 ; * \star P=0.01 \sim 0.001 ; * \star \star P \otimes 0.001$.

\section{Supplementary Files}

This is a list of supplementary files associated with this preprint. Click to download.

- SupplementaryTable.xlsx 